\title{
Study of varieties of spring triticale for the presence of the wbm gene
}

\author{
Kolenkov M. ${ }^{1 *}$, Ozherelev P. ${ }^{3}$, Gruzdev I. ${ }^{1}$, Soloviev A. ${ }^{1}$, Kirov I. ${ }^{1,2}$ \\ ${ }^{1}$ Laboratory of Marker-Assisted and Genomic Selection of Plants, All-Russia Research Institute \\ of Agricultural Biotechnology, Moscow, Russia \\ ${ }^{2}$ Kurchatov Genomic Center of ARRIAB, All-Russia Research Institute of Agricultural Biotechnology, \\ Moscow, Russia \\ ${ }^{3}$ Russian State Agrarian University - Moscow Timiryazev Agricultural Academy, Moscow, Russia \\ * email: colenckov@yandex.ru
}

Baking qualities are the most important characteristic of many main grain crops, on which the quality of future bread directly depends. The genes that determine these qualities have not yet been adequately studied, so we have an incomplete understanding of the inheritance of this characteristic and the mechanisms that lead to an increase in baking qualities. A gene called wbm (for wheat bread making) has been identified in wheat and has been shown to be expressed in increased levels in the endosperm during caryopsis development. We proved that wbm is localized on chromosome 7AL and demonstrated significant diversity in the wbm protein sequence between closely related species. The location of wbm in genome A provides an opportunity for screening a collection of hexaploid triticale lines (AABBRR, $2 n=6 x=42$ ) and allowed us to identify three triticale lines carrying this gene. These results allowed us to include the lines of spring triticale carrying the wbm gene in the scheme of breeding crosses. It is assumed that the analysis of the progeny of crosses will allow us to accurately determine the function of the wbm gene protein and determine the pattern of gene inheritance. 